\title{
ANÁLISE DA EXPOSIÇÃO À VIBRAÇÃO DE CORPO INTEIRO EM HARVESTERS DE ESTEIRAS E PNEUS NA OPERAÇÃO DE DESBASTE
}

\author{
Alysson Braun Martins ${ }^{1 *}$, Felipe Martins de Oliveira ${ }^{1}$ \\ ${ }^{1}$ Laboratório de colheita e transporte florestal, Universidade Estadual do Centro-Oeste, 84500000, Irati, Brasil.
}

*E-mail: alyssonbmartins@gmail.com

Recebido em:19/04/2019 Aceito em:05/07/2019

\section{RESUMO}

No Brasil, pouco são os estudos relacionados a exposição ocupacional à vibração de corpo inteiro em postos de trabalho de máquinas florestais e, quando consideradas operações de desbaste, não há estudos relacionados. Este estudo foi realizado na região de Bituruna-Pr, em povoamentos de pinus taeda com 10 anos de idade submetidos ao primeiro desbaste, objetivando quantificar os níveis de vibração a que estão expostos os operadores de dois tipos de harvester - esteiras e pneus - durante a jornada de trabalho. Utilizouse um medidor integrador triaxial Bruel e kjaer modelo Type 4447 e os resultados analisados conforme os critérios estabelecidos pela NHO 09. O harvester de esteiras apresentou resultados de aren e VDVR equivalentes a $0,6 \mathrm{~m} / \mathrm{s}^{2}$ e $11,2 \mathrm{~m} / \mathrm{s}^{1,75}$ respectivamente, enquanto o harvester de pneus apresentou valores médios de $0,4 \mathrm{~m} / \mathrm{s}^{2}$ e $9,3 \mathrm{~m} / \mathrm{s}^{1,75}$. Os resultados indicaram diferenças significativas pelo teste $\mathrm{t}(\mathrm{p}<0,05)$, indicando, portanto, melhores condições de trabalho no posto de trabalho do harvester de pneus. Apesar da significativa modernização empregada em máquinas de colheita florestal até os dias atuais, observa-se que a exposição ocupacional à vibração ainda é um fator ergonômico que pode comprometer o conforto, saúde e segurança de operadores dessas máquinas e, quando comparadas máquinas purpose built com máquinas adaptadas, os riscos à saúde são ainda maiores.

Palavras-chave: Vibração. Harvester. Desbaste.

\section{Introdução}

O segmento de florestas plantadas no Brasil cresceu significativamente nas últimas décadas, sendo o impulso inicial a partir da década de 1970, com a intensificação dos plantios de espécies exóticas como Eucalyptus e Pinus. Aliado ao crescimento do setor de florestas plantadas, o processo de mecanização da colheita de madeira em larga escala também foi intensificado, com incremento a partir da década de 1990 ocasionado pela abertura do mercado às importações de máquinas de elevada tecnologia. Entretanto, apesar da notória evolução tecnológica em máquinas florestais, e consequente evolução em termos de conforto e segurança dos operadores, fatores ergonômicos como a exposição ocupacional à vibração ainda podem influenciar negativamente no posto de trabalho dessas máquinas.

Atualmente, observa-se nas empresas florestais brasileiras que há dois segmentos de máquinas florestais: as máquinas importadas (purpose built), as quais foram projetadas exclusivamente para fins florestais; e máquinas adquiridas no mercado nacional (máquinas base com implemento florestal). Com isso, operações como o corte e processamento de madeira realizado pelo harvester, podem sofrer variações ergonômicas em termos de exposição ocupacional à vibração. E, quando realizadas em regime de desbaste, que proporciona baixa produtividade e maior mobilidade, as variações podem ser ainda mais significativas.

A exposição à vibração de corpo inteiro pode causar danos físicos permanentes ou distúrbios no sistema nervoso, sendo que, a exposição diária pode resultar em danos na região espinhal e afetar os sistemas circulatório e urológico. Alguns sintomas de distúrbios geralmente aparecem durante a exposição sob a forma de fadiga, insônia, dor de cabeça e tremor [1].

Realizou-se uma avaliação quantitativa da exposição à vibração em operadores de harvester, slingshot, fellerbuncher, e forwarder em situações habituais de trabalho, com o intuito de avaliar os potenciais efeitos da exposição à vibração de corpo inteiro, bem como as condições de segurança no posto de trabalho. Com base nos resultados, a autora concluiu que, com exceção do eixo $\mathrm{x}$ do feller-buncher e eixo y do forwarder, todos os outros dados encontrados estavam acima do imite estabelecido na norma ISO 2631-1: 1985, a qual foi usada como referência [2].

Avaliou-se a exposição ocupacional à vibração de corpo inteiro em máquinas de colheita florestal, e concluíram que a atividade laboral do operador de máquinas florestais tem 
como principal agravante de problemas ocupacionais a vibração recebida por aproximadamente $75 \%$ da jornada diária, agravando-se pelo elevado tempo em mesma postura (sentada) [3].

Realizou-se um estudo para avaliar ergonomicamente o ambiente de trabalho de operadores de harvester e forwarder. Para a seleção dos fatores a serem estudados, os autores realizaram uma pesquisa bibliográfica, na qual identificaram a exposição ocupacional à vibração como fator relevante para o estudo. Os autores identificaram níveis médios de vibração em $0,46 \mathrm{~m} / \mathrm{s}^{2}$ [4].

Os limites e recomendações acerca da exposição às vibrações estão previstos em normas internacionais, as quais servem de referência para a legislação nacional pertinente ao assunto [4].

A exposição às vibrações mecânicas é um importante fator a que operadores de máquinas florestais estão sujeitos e, se tratando de máquinas que possuem diferentes características de projeto, acredita-se que os níveis de viração podem ser significativamente distintos, principalmente quando operadas em regime de desbaste.

Em função disso, este trabalho buscou quantificar os níveis de exposição à vibração em que operadores de harvester, com rodados de esteiras e pneus, estão sujeitos na operação de desbaste e, a partir dos dados obtidos, comparar com as recomendações previstas na norma vigente.

\section{Metodologia}

Este estudo foi realizado em duas áreas de colheita de pinus taeda, de outubro de 2018 a janeiro de 2019, em uma empresa florestal localizada em Bituruna-PR. A avaliação quantitativa envolveu dois operadores, sendo um para o harvester de pneus e um para o harvester de esteiras, os quais foram escolhidos conforme experiência na função e produtividade média em relação à meta estabelecida pela empresa. Os operadores estudados tiveram participação voluntária, recebendo esclarecimentos quanto aos objetivos da pesquisa, por meio da assinatura do Termo de Consentimento Livre e Esclarecido (TCLE), em atendimento à Resolução n. 466/2012 do Conselho Nacional de Saúde do Ministério da Saúde [5].

A operação foi realizada em povoamentos de pinus taeda submetidos ao primeiro desbaste com dez anos de idade e volume médio individual com casca de $0,289 \mathrm{~m}^{3}$. As áreas de estudo apresentaram declividades até $15^{\circ}$, identificados pelo computador de bordo das máquinas. Os harvesters avaliados no estudo são caracterizados conforme a tabela 1 .
Tabela 1 - Caracterização das máquinas avaliadas

\begin{tabular}{ccccccc}
\hline Harv. & $\begin{array}{c}\text { Pot. } \\
(\text { HP) }\end{array}$ & $\begin{array}{c}\text { Peso } \\
\text { (ton) }\end{array}$ & $\begin{array}{c}\text { Larg. } \\
(\mathbf{m m})\end{array}$ & $\begin{array}{c}\text { Alt. } \\
(\mathbf{m m})\end{array}$ & $\begin{array}{c}\text { Alc. } \\
(\mathbf{m})\end{array}$ & $\begin{array}{c}\text { Hor. } \\
\text { (horas) }\end{array}$ \\
\hline Pneus & 255 & 20,7 & 2.990 & 3.930 & 10 & 12.000 \\
Esteiras & 115 & 13,9 & 2.600 & 3.110 & 10 & 13.000 \\
\hline
\end{tabular}

Para a avaliação da exposição de corpo inteiro, adotou-se a norma NHO 09, a qual estabelece como critério de avaliação, os valores de vibração recebidos nos três eixos ortogonais (x, y e z). Para tal, a norma utiliza os valores de Aceleração Resultante da Exposição Normalizada (aren) e Valor da Dose de Vibração Resultante (VDVR), calculados de forma ponderada, sendo os eixos $\mathrm{x}$ e $\mathrm{y}=1,4$; e eixo $\mathrm{z}=1,0$ [6].

aren $=$ are $\sqrt{\frac{T}{T_{0}}} \quad\left[\mathrm{~m} / \mathrm{s}^{2}\right]$

$\operatorname{VDVR}=\left[\Sigma_{j}\left(\text { VDVexp }_{j}\right)^{4}\right]^{1 / 4}$

A partir dos valores de aren e VDVR encontrados, a norma sugere níveis de ação e as respectivas recomendações conforme a Tabela 2, sendo o nível de ação, valor no qual são indicadas medidas preventivas e, limite de exposição, valor no qual são indicadas medidas corretivas.

Tabela 2 - Critérios de avaliação pela NHO 09

\begin{tabular}{|c|c|c|c|}
\hline $\begin{array}{c}\operatorname{aren} \\
\left(\mathrm{m} / \mathbf{s}^{2}\right)\end{array}$ & $\begin{array}{l}\text { VDVR } \\
\left(\mathrm{m} / \mathbf{s}^{1,75}\right)\end{array}$ & $\begin{array}{c}\text { Consideração } \\
\text { Técnica }\end{array}$ & Recomendação \\
\hline 0 a 0,5 & 0 a 9,1 & Aceitável & $\begin{array}{c}\text { Manutenção da } \\
\text { condição existente }\end{array}$ \\
\hline $\begin{array}{l}0,5 \mathrm{a} \\
<0,9\end{array}$ & $\begin{array}{c}>9,1 \mathrm{a}< \\
16,4\end{array}$ & $\begin{array}{l}\text { Acima do nível } \\
\text { de Ação }\end{array}$ & $\begin{array}{c}\text { Adoção de medidas } \\
\text { preventivas }\end{array}$ \\
\hline 0,9 a 1,1 & $\begin{array}{c}16,4 \mathrm{a} \\
21,0\end{array}$ & $\begin{array}{l}\text { Região de } \\
\text { Incerteza }\end{array}$ & $\begin{array}{c}\text { Adoção de medidas } \\
\text { preventivas e } \\
\text { corretivas visando a } \\
\text { redução da } \\
\text { exposição diária }\end{array}$ \\
\hline $\begin{array}{l}\text { Acima } \\
\text { de } 1,1\end{array}$ & $\begin{array}{c}\text { Acima de } \\
21,0\end{array}$ & $\begin{array}{l}\text { Acima do limite } \\
\text { de Exposição }\end{array}$ & $\begin{array}{l}\text { Adoção imediata de } \\
\text { medidas corretivas }\end{array}$ \\
\hline
\end{tabular}

Os níveis de vibração foram obtidos a partir de um medidor de vibração triaxial da marca Bruel e kjaer, modelo Type 4447 que atende os parâmetros estabelecidos na NHO 09. Esse equipamento fornece a soma de cada eixo de forma integrada e a somatória total da aceleração encontrada. O acelerômetro foi fixado no assento das máquinas, através de um dispositivo almofadado com um acelerômetro acoplado e o aparelho fixado na cintura do operador (Figura 1). 


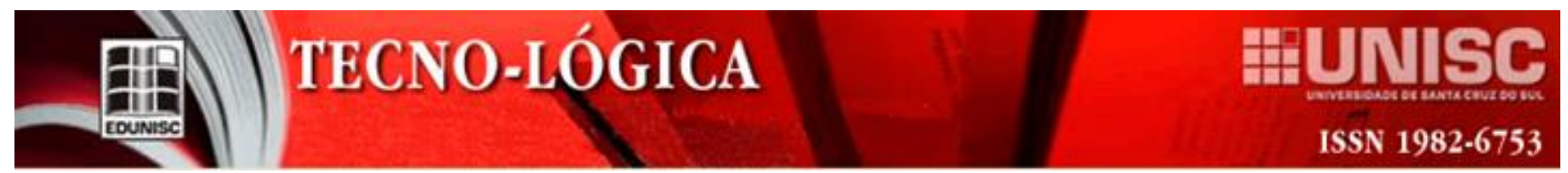

dados durante 30 minutos por amostra realizada, de forma a
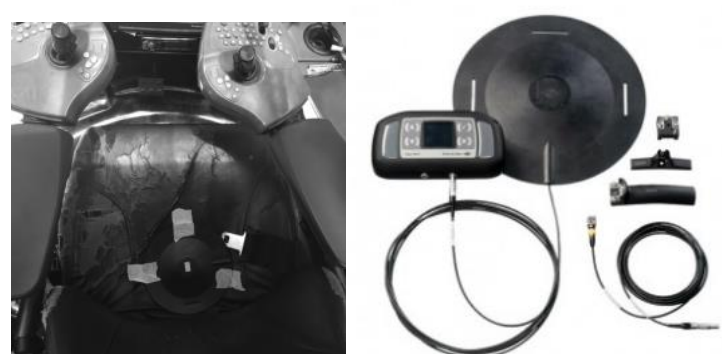

Figura 1 - Medidor de vibração Bruel e kjaer - Type 4447; Posicionamento da almofada no assento.

A fim de se obter um número significativo de amostras, inicialmente foi realizado um estudo piloto [7], para se obter um número mínimo de amostras a serem realizadas com $95 \%$ de confiança.

$\mathrm{n} \geq \frac{\mathrm{t}^{2} * \mathrm{~s}^{2}}{\mathrm{e}^{2}}$

Em que: $\mathrm{n}=$ número de amostras necessárias; valor tabelado a 95\% de probabilidade (Distribuição t de Student); s = Desvio padrão; e e = Erro Admissível.

Dessa forma, as medições foram realizadas no decorrer de vinte e quatro dias, sendo que foram registrados cobrir um número aceitável de ciclos operacionais.

A fim de avaliar comparativamente a exposição ocupacional à vibração nas duas máquinas, os resultados foram submetidos ao teste de comparação de médias de student (teste t), visando comprovar estatisticamente se os resultados são significativamente diferentes.

\section{Resultados e discussões}

A partir da análise de dados, identificou-se os resultados da aren e VDVR conforme apresentados na Tabela 2. Os resultados do teste $\mathrm{t}$ são apresentados na tabela 3 . Observa-se que os níveis de vibração em que o operador do harvester de esteiras estava exposto ficou acima do nível aceitável para o trabalho. $\mathrm{O}$ resultado da aren apresentou valor médio acima do nível da ação $\left(0,6 \mathrm{~m} / \mathrm{s}^{2}\right)$, demonstrando que a máquina de esteiras proporcionou níveis de vibração acima do ideal para o trabalho A NHO 09 indica que, níveis de aren entre 0,5 e $1,1 \mathrm{~m} / \mathrm{s}^{2}$, requerem medidas preventivas no posto de trabalho, a fim de se evitar que os níveis de vibração ultrapassem os valores toleráveis e possam ocasionar problemas a saúde do trabalhador. Esta situação não foi identificada no harvester de pneus, pois a média da aren encontrada foi equivalente a $0,4 \mathrm{~m} / \mathrm{s}^{2}$, valor abaixo do nível de ação proposto pela norma.

\begin{tabular}{|c|c|c|c|c|c|c|c|c|c|c|c|}
\hline \multirow{3}{*}{ Máquina } & \multicolumn{2}{|c|}{$\begin{array}{l}\text { Média do ciclo } \\
\text { operacional (seg) }\end{array}$} & \multicolumn{3}{|c|}{ Acelerações médias $\left(\mathbf{m} / \mathbf{s}^{2}\right)$} & \multirow{3}{*}{$\begin{array}{c}\text { Média } \\
\text { da aren } \\
\left(\mathbf{m} / \mathbf{s}^{2}\right)\end{array}$} & \multirow{3}{*}{$\begin{array}{c}\text { Média do } \\
\text { VDVR } \\
\left(\mathbf{m} / \mathbf{s}^{1,75}\right)\end{array}$} & \multicolumn{4}{|c|}{ NHO 09} \\
\hline & & & \multirow[b]{2}{*}{ Eixo X } & \multirow[b]{2}{*}{ Eixo Y } & \multirow[b]{2}{*}{ Eixo Z } & & & \multicolumn{2}{|c|}{ aren } & \multicolumn{2}{|c|}{ VDVR } \\
\hline & & & & & & & & NA & $\mathbf{L E}$ & NA & $\mathbf{L E}$ \\
\hline \multirow{4}{*}{ Esteiras } & & 37 & 0,383 & 0,276 & 0,370 & & & \multirow{6}{*}{0,5} & \multirow{6}{*}{1,1} & \multirow{6}{*}{9,1} & \multirow{6}{*}{21,0} \\
\hline & $\mathrm{s}$ & 2,1 & 0,197 & 0,152 & 0,227 & 0,6 & 11,2 & & & & \\
\hline & $\mathrm{cv}$ & $5,6 \%$ & $59,8 \%$ & $65,9 \%$ & $77,8 \%$ & & & & & & \\
\hline & & 32 & 0,175 & 0,187 & 0,151 & \multirow{3}{*}{0,4} & \multirow{3}{*}{9,3} & & & & \\
\hline \multirow[t]{2}{*}{ Pneus } & $\mathrm{s}$ & 5,0 & 0,100 & 0,123 & 0,064 & & & & & & \\
\hline & $\mathrm{cv}$ & $15,6 \%$ & $57,4 \%$ & $65,9 \%$ & $42,1 \%$ & & & & & & \\
\hline
\end{tabular}

$\mathrm{s}=$ desvio padrão; $\mathrm{cv}=$ coeficiente de variação; $\mathrm{NA}=$ nível de ação; $\mathrm{LE}=$ limite de exposição

Quando analisado o VDVR, observa-se que ambas as máquinas obtiveram valores acima do nível de ação, com valores de 11,2 e $9,3 \mathrm{~m} / \mathrm{s}^{1,75}$, para o harvester de esteiras e o de pneus respectivamente, indicando a necessidade de adoção de medidas preventivas em ambos os postos de trabalho. $\mathrm{O}$
VDVR utiliza para a análise um método mais sensível de análise da exposição à vibração - elevado a quarta potência sendo indicado para a análise de picos de vibração. Visto isso, o VDVR está relacionado aos choques ou solavancos que o operador ficou exposto no posto de trabalho. 
Aceleração Resultante de Exposição Normalizada (aren)

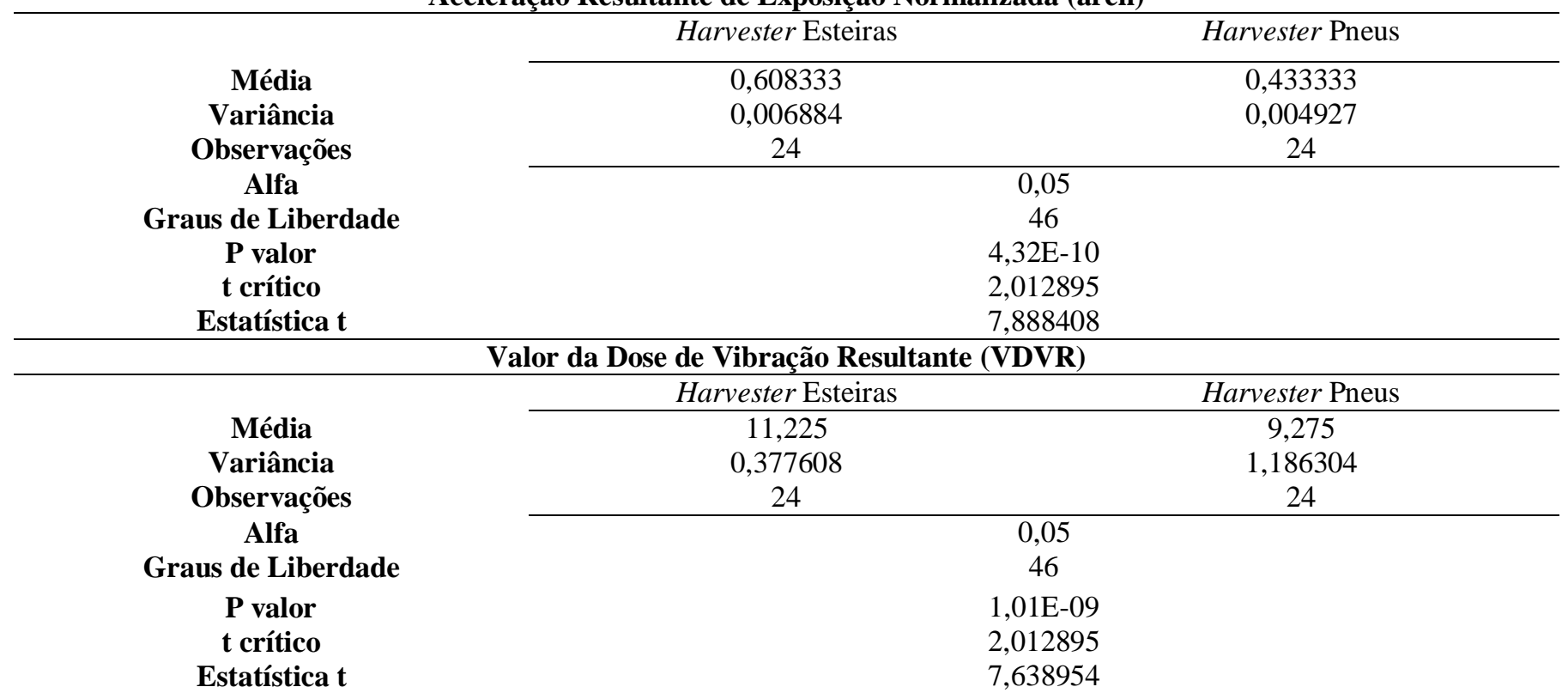

Devido ao corte e processamento da madeira ocorrer em espaços extremamente reduzidos, o contato da grua das máquinas com as árvores, muita das vezes se torna inevitável, gerando os picos de vibração momentâneos. Outro fator que pode ser atribuído aos picos de vibração é o deslocamento da máquina que, devido a irregularidade do terreno - presença de galhos e tocos - gera solavancos no posto de trabalho. Destaca-se ainda que, devido às características de projeto das máquinas, notou-se durante a operação que o harvester de pneus suportou melhor os impactos sofridos, podendo estar relacionados com o sistema de nivelamento e amortecimento da cabine.

A análise estatística pelo teste $\mathrm{t}(\mathrm{p}<0,05)$ demonstrou que os valores de aren e VDVR apresentaram-se significativamente diferentes entre o harvester de esteiras e pneus, indicando condições inferiores para a máquina de esteiras em termos de exposição ocupacional à vibração. A diferença significativa entre as máquinas pode ser atribuída principalmente às características de projeto das máquinas pois, devido ao harvester de esteiras não ter sido desenvolvido para fins florestais, ou seja, não é "purpose built", quando operada nessa condição, o sistema de atenuação da vibração emitida se torna ineficiente. Por outro lado, o harvester de pneus suporta melhor as condições operacionais, proporcionando melhor conforto não somente em termos de exposição à vibração, mas também outros fatores ergonômicos.

Outro fator a ser destacado, refere-se ao fato de o assento do harvester de pneus estar em melhores condições de conservação. Tal observação foi confirmada pelos operadores que relataram a percepção de melhores condições de conforto na máquina de pneus. Acredita-se que essa condição pode ter contribuído para os níveis elevados de vibração, pois o assento atua como atenuante da vibração gerada pela máquina antes do contato com o operador.

Gerasimov e Sokolov [8], realizaram uma análise ergonômica comparativa entre 13 máquinas de colheita florestal (Havester, Forwarder, Feller-buncher e Skidder) e, dentre os aspectos observados, avaliaram a exposição ocupacional à vibração de corpo inteiro como um dos fatores que influenciam no desempenho do operador. Com base na metodologia proposta, os autores identificaram que o harvester apresenta as melhores condições ergonômicas em termos de vibração, de acordo com a norma avaliada.

Gerasimov e Sokolov [9], realizaram uma avaliação ergonômica comparativa entre 14 sistemas de colheita de madeira a fim de identificarem quais conjuntos de máquinas são menos prejudiciais à saúde dos operadores. Dentre os fatores avaliados, a vibração foi medida em sete diferentes modelos de harvester. Os autores obtiveram valores de aceleração média equivalente a $0,3 \mathrm{~m} / \mathrm{s}^{2}$, indicando conformidade com os valores estabelecidos nas normas avaliadas.

Sherwin et al. [10], avaliaram a influência da pressão de enchimento dos pneus nas vibrações de corpo inteiro transmitidas ao operador de harvester, e encontraram valores similares ao deste estudo, com valores de aren e VDVR equivalentes a 0,56 e 10,22 respectivamente com pressão dos pneus em $414 \mathrm{kPa}$. Entretanto, destaca-se nos resultados que, quando a pressão dos pneus foi reduzida para $345 \mathrm{kPa}$, os valores de aren e VDVR reduziram para 0,38 e 6,96 respectivamente, demonstrando que redução de pressão dos 
pneus pode ser um importante atenuante para exposição ocupacional à vibração em máquinas florestais.

Marzano et al. [11], realizaram análise comparativa entre as máquinas florestais harvester e Forwarder e, dentre os fatores de avaliação, identificaram níveis de vibração entre 0,27 e $0,70 \mathrm{~m} / \mathrm{s}^{2}$ na colheita de madeira de eucalipto. Os resultados identificados pelos autores estão de acordo com níveis de aren encontrados no presente estudo, demonstrando que as operações de colheita florestal com o harvester apresentam níveis de vibração próximos do nível de ação $\left(0,5 \mathrm{~m} / \mathrm{s}^{2}\right)$.

Jankovsky et al. [4], realizaram um estudo para avaliar ergonomicamente o ambiente de trabalho de operadores de harvester e forwarder e, dentre as máquinas avaliadas, 3 eram harvesters e 2 Forwarders, ambos com rodados de pneus na colheita de pinus. Os autores identificaram valores de aren médios em $0,46 \mathrm{~m} / \mathrm{s}^{2}$, valor próximo ao encontrado nesse estudo. Nota-se que as condições de operação são semelhantes, justificando-se os resultados similares.

\section{Conclusões}

Conclui-se que a atividade laboral do operador de máquina florestal é uma tarefa complexa, demandando um longo período em uma mesma postura (sentada) e sendo agravado pela exposição à vibração recebida durante grande parte da jornada de trabalho. Considerando as características operacionais da colheita de madeira em povoamentos submetidos ao desbaste, os problemas ocupacionais podem ser ainda maiores. Os níveis de vibração nos postos de ambas as máquinas estudadas estavam em condições de conformidade com a NHO 09, porém, sendo recomendada a adoção de medidas preventivas a fim de reduzir a aren e o VDVR para valor abaixo do nível de ação proposto.

A indústria de máquinas florestais tem intensificado o desenvolvimento e aplicação de inovações tecnológicas, buscando proporcionar melhorias em termos de conforto e segurança e consequente produtividade dos operadores. No entanto, há de se destacar que esse parâmetro deve caminhar em conjunto com os aspectos organizacionais, pois, é primordial que as máquinas tenham elevada tecnologia embarcada, mas também haja limitações para as jornadas de trabalho, bem como pausas e exercícios laborais. Além disso, destaca-se como principal fator que interfere nos aspectos ergonômicos de postos de trabalho de máquinas florestais, o projeto das máquinas, pois atribui-se como principal agravante máquinas que não são desenvolvidas para fins florestais.
Agradecimentos

Agradecemos à CAPES, pelo apoio e financiamento deste estudo.

\section{COMPARATIVE ANALYSIS OF THE OCCUPATIONAL EXPOSURE TO HARVESTER VIBRATION OF TEARS AND TIRES IN PINUS DESBASTE}

\begin{abstract}
In Brazil, there are few studies related to occupational exposure to full body vibration in forestry machine workstations and, when considered thinning operations, there are no related studies. This study was carried out in the region of Bituruna-Pr, in 10-year-old pinus taeda stands subjected to the first thinning, aiming to quantify the vibration levels to which operators of two types of harvester treadmills and tires - are exposed during the working day. A Bruel and kjaer type 4447 triaxial integrator meter was used and the results were analyzed according to the criteria established by NHO 09. The track harvester presented results of aren and VDVR equivalent to $0.6 \mathrm{~m} / \mathrm{s}^{2}$ and $11.2 \mathrm{~m} / \mathrm{s}^{1.75}$ respectively, while the tire harvester presented mean values of $0.4 \mathrm{~m} / \mathrm{s}^{2}$ and $9.3 \mathrm{~m} / \mathrm{s}^{1.75}$. The results indicated significant differences by the $t$ test $(\mathrm{p}<0.05)$, indicating, therefore, better working conditions at the tire harvester workstation. Despite the significant modernization used in forest harvesting machines to the present day, it is observed that occupational exposure to vibration is still an ergonomic factor that can compromise the comfort, health and safety of operators of these machines and, when compared machines purpose built with machines, health risks are even greater.
\end{abstract}

Keywords: Vibration. Harvester. Thinning

\section{Referências}

[1] SAliBA, T. M. Manual Prático de Avaliação e Controle de Vibração PPRA. São Paulo. LTr. 2016. 112p

[2] YAMASHITA, R. Y. Avaliação das condições de trabalho e da exposição à vibração do operador de máquinas na colheita florestal. 2002. 55 p. Dissertação (Mestrado em Ciências Florestais) - Escola Superior de Agronomia "Luiz de Queiroz", Piracicaba, 2002.

[3] AlmEIDA, S. F.; ABRAHÃO, R. F.; TERESO, M. J. A. Avaliação da exposição ocupacional à vibração de corpo inteiro em máquinas de colheita florestal. Revista Cerne, Campinas, v. 21, n. 1, p. 1-8, 2015.

[4] JANKOVSKY, M.; MESSINGEROVA, V.; FERENEIK, M.; ALLMAN, M. Objective and subjective assessment of selected factors of the work environment of forest harvesters and forwarders. Journal of Forest Science, 2016;62(1);8-16. 
[5] BRASIL. Conselho Nacional de Saúde. Resolução $\mathrm{n}^{\circ}$ 466, de 12 de dezembro de 2012. Aprova normas regulamentadoras de pesquisas envolvendo seres humanos. Brasília: Diário Oficial da União, 13 jun. 2013 b. Seção I, p. 59.

[6] FUNDAÇÃO JORGE DUPRAT FIGUEIREDO DE SEGURANÇA E SAÚDE NO TRABALHO. Norma de Higiene Ocupacional. NHO 09: Procedimento Técnico: Avaliação da Exposição Ocupacional a Vibrações de Corpo Inteiro. São Paulo, p. 40. 2013.

[7] CONAW, P. L. Estatística. São Paulo: E. Blucher, 1977. 264 p.

[8] GERASIMOV Y., SOKOLOV A. Ergonomic characterization of harvesting work in Karelia. Croatian Journal of Forest Engineering, 2009.

[9] GERASIMOV, Y.; SOKOLOV, A. Ergonomic evaluation and comparison of wood harvesting systems in Northwest Russia. Applied Ergonomics, v.45, 318-38, 2014

[10] SHERWIN, L. M.; OWENDE, P. M. O.; KANALI, C. L.; LYONS, J.; WARD, S. M. Influence of tyre inflation pressure on whole-body vibrations transmitted to the operator in a cut-to-length timber harvester. Applied Ergonomics. 2004; 35: 253-261

[11] MARZANO, F. L. C.; SOUZA, A. P.; MINETTE, L. J. Proposal for an ergonomic conformity index for evaluation of harvesters and forwarders. Revista Árvore. 2017; 41(4):e410401. 